\title{
Solving an optimal control problem of hepatitis B virus dynamics: Efficacy of fuzzy logic strategy
}

\author{
Marcel Gahamanyi ${ }^{1}$, Wellars Banzi ${ }^{1}$ and Jean Marie Ntaganda ${ }^{1}$, \\ ${ }^{1}$ University of Rwanda, College of Science and Technology, School of Science, Department of Mathematics \\ *Corresponding Author: j.m.ntaganda@ur.ac.rw, jmnta@yahoo.fr \\ DOI: $10.4314 /$ rjeste.v4i1.1 \\ https://dx.doi.org/10.4314/rjeste.v4i1.1
}

\begin{abstract}
This work aims at using fuzzy logic strategy to solve a hepatitis B virus (HBV) optimal control problem. To test the efficacy of this numerical method, we compare numerical results with those obtained using direct method. We consider a patient under treatment during 12 months where the two drugs are taken as controls. The results are rather satisfactory. In particular, the reaction of HBV to drugs can be modeled and a feedback can be approximated by the solution of a linear quadratic problem. The drugs reduce the risk of HBV. Furthermore, results of both numerical methods are in good agreement with experimental data and this justifies the efficacy of fuzzy logic strategy in solving optimal control problems.
\end{abstract}

Mathematics Subject classifications (MSC 2010): 03B52, 93C15, 49J15, 41A58

Keywords: Hepatitis B virus, Mathematical model, Optimal control, Direct method, Fuzzy logic strategy, Membership degree, Numerical test

\section{Introduction}

Hepatitis B is one of diseases transmitted through contact either with blood or bodily fluids from an individual infected with the hepatitis B virus (HBV). It attacks an important organ of human body, the liver. Therefore, it called liver infection since the virus mainly affects liver function by invading the liver cells (hepatocytes) and uses the cells' machinery to replicate within it. HBV results in different and serious liver diseases such as chronic hepatic insufficiency, hepatocellular carcinoma, cirrhosis and can be a potential cause of the liver cancer. According to The World 
Health Organisation (WHO), HBV is in two possible phases [21]: Acute hepatitis B infection that lasts less than six months and chronic hepatitis B infection which lasts six months or longer. There are more than 350 million people living with hepatitis B virus infection, most of which resulted in more than 0.8 million deaths per year due to HBV-related liver disease and liver cancer. The age can be considered to be a factor of developing a chronic infection in the sense that, children less than 6 years of age who become infected with the HBV are the most likely to develop chronic infection [22].

To date, the way to eliminate HBV transmission is to immunise susceptible individuals, especially newborns. Although process of immunisation brings to success, the prevalence prediction of long term in HBV remains challenge to provide useful information for public health decision-making. The mathematical model use is one feasible method to predict the prevalence of infectious disease including HBV [24]. These models can be one useful tools in controlling hepatitis B virus in order to put down the infection from the population.

The transmission dynamics model or the compartmental model is an important theoretical epidemiology method used to study the transmission dynamics of infectious diseases. The transmission dynamics model was first used in the early 1980s to study the transmission dynamics of hepatitis B and the effectiveness of control [13]. Some of these mathematical models address questions concerning the impact of vaccination and others have given a quantitative basis for making optimal decisions in public health policy regarding HBV transmission [6]. Anderson and May used a simple mathematical model to illustrate the effects of carriers on the transmission of HBV [1]. The mathematical model developed by Pang et al. [16] allowed them to explore the impact of vaccination and other controlling measures of HBV infection while Bhattacharyya and Ghosh [2], Kar and Batabyal [8], and Kar and Jana [9] proposed optimal control of infectious diseases. The optimal control theory has found wideranging applications in biological and ecological problems [12]. The use of this theory has been designed using model predictive control (MPC) method [5].

Motivated by the works mentioned above, we present in this work the method of using fuzzy logic strategy [14] to solve an optimal control problem of an uninfected Hepatitis B virus dynamics. To test its efficacy, we compare the solutions from this strategy with one obtained using direct method. It could be possible that one can use the same method to solve the similar problem that is related to different type of hepatitis such as A or C [15]. The contribution of this 
paper comes in the formulation of HBV dynamics and control strategies and solving the resulting optimal problem for Hepatitis B virus. The other sections of this paper are organized as follows. The section 2 presents the methods and material. The mathematical model equations, optimal control problem, and fuzzy logic strategy as well as direct method are presented this this section. The section 3 deals with discretization of optimal control problem using fuzzy logic strategy and direct method. The numerical tests from simulations are presented in section 4. Finally, the section 5 deals with concluding remarks.

\section{Methods and material}

\subsection{Governing equations}

The mathematical model describing HBV dynamics is formulated as a system of three ordinary differential equations [5]. The dependent variables are T, I and V. At time t, T denotes the concentration of uninfected hepatocytes, I is the concentration of infected hepatocytes and V represents the concentration of free virions. The governing equations read

$$
\begin{aligned}
& \frac{d T}{d t}=s-q T+a T\left[1-\frac{T}{T_{\max }}\right]-\beta e^{-u_{1}} \frac{T V}{1+b V}, \\
& \frac{d I}{d t}=\beta e^{-u_{1}} \frac{T V}{1+b V}-\delta I, \\
& \frac{d V}{d t}=p e^{-u_{2}} I-c V,
\end{aligned}
$$

where $\mathrm{s}$ and $\mathrm{qT}$ are constant rate of production and death rate of uninfected hepatocyte, respectively. Tmax (cells/dl) is the $T$ concentration at which proliferation shuts off. The constant a stands for the maximum proliferation rate of uninfected hepatocyte cells. This proliferation is modeled using logistic function [4] such that the maximum concentration of uninfected hepatocyte cells shuts off. The rate of infection is given by saturation functional response $\beta e^{-u_{1}} T V /(1+b V)$, where $\beta$ denotes the constant infection rate which characterizes the infection efficiency and where $b$ is a positive constant. The term $\delta I$ denotes death rate of infected hepatocytes. The free virions are produced from infected hepatocytes at the rate of $p e^{-u_{2}} I$ with $c V$ taken as clearance rate of viral particles. The mathematical model (1)-(3) shows the role of therapy. Furthermore, the first type of drug is described by chemotherapy 
functions $e^{-u_{1}}$. It helps to prevent the virus from infecting the cell and the second denoted by the function $e^{-u_{2}}$ facilitates the prevention of the infected cells from producing the new viruses. The values of initial conditions of the mathematical model (1)-(3) are presented in section numerical test below.

\subsection{Optimal control problem}

To formulate optimal control problem, let $E=(T, I, V)^{t}$ be a state vector and $E_{0}=\left(T_{0}, 0,0\right)^{t}$ denotes an uninfected steady state vector for healthy human. The constant $T_{0}$ stands for concentration at which uninfected hepatocyte cells are found out. According to physiological theory of HBV the cost functional can be formulated as follows [3],

$J\left(u_{1}, u_{2}\right)=\int_{0}^{T_{f}} q_{T}\left(T-T_{0}\right)^{2}+q_{u_{1}} u_{1}(t)^{2}+q_{u_{2}} u_{2}(t)^{2}$

The positive scalar coefficients $q_{T}, q_{u_{1}}$ and $q_{u_{2}}$ are weights attached to each cost component term whereas $\mathrm{T}_{\mathrm{f}}$ denotes the maximum time of prescription drug. The purpose is to minimize this objective function subject to the system (1)-(3).

\subsection{Fuzzy logic strategy}

We are interested in describing briefly an approach for solving mathematical problem developed in1985 by Takagi-Sugeno [19], [20]. This approach uses a following general discrete optimization problem we want to be interested in. Furthermore, we want to find $U_{k}^{*} \in R^{N}$, $k=0, \ldots, N-1$ that minimizes

$$
J\left(U_{0}, \ldots, U_{N-1}\right)=\sum_{k=0}^{N-1}\left(x_{k}^{T} R x_{k}+U_{k}^{T} Q U_{k}\right),
$$

subject to

$\left\{\begin{array}{l}x_{k+1}=f_{k}\left(x_{k}, U_{k}\right) \\ x_{k} \in R^{N}, U_{k} \in R^{N}\end{array}, k=0, \ldots, N-1\right.$

where $R$ and $Q$ are positive defined matrices, $\mathrm{x}_{\mathrm{k}}$ is state element and $\mathrm{f}_{\mathrm{k}}$ : $\mathrm{R}^{\mathrm{N}} \times \mathrm{R}^{\mathrm{N}} \rightarrow \mathrm{R}^{\mathrm{N}}$ denotes a discrete function. This problem can also be solved using the dynamic programming method. However, the rate of convergence of this method is quadratic and solution is often represented as 
a state of control feedback [16] and this solution which in some cases is not optimal depends on the choice of the initial trajectory. The mathematical problem is carried out to find fuzzy linearization regions in the state [17]. Taking these fuzzy regions as basis, non linear system is decomposed in a structured multi models of several independent linear models [10]. The linearization is made around an operating point contained in these regions.

Let $x_{i}, i=1, \ldots, S$ be operating point among total $\mathrm{S}$ points and $N L(x)$ stands for a nonlinear term of a mathematical model. There are two kinds of estimates of $N L(x)$ :

$N L(x) \approx N L_{0}(x)=N L\left(x_{i}\right)$,

and

$N L(x) \approx N L_{1}(x)=N L\left(x_{i}\right)+\left(\frac{d N L(x)}{d x}\right)\left(x-x_{i}\right)$.

To minimize the error between the non linear function and the fuzzy approximation, TakagiSugeno introduced factor of the consequence for fuzzy [19]. Let $\varepsilon$ denotes this factor, the approximation (8) can be formulated as follows:

$$
\begin{aligned}
N L(x) & \approx(1-\beta) N L_{0}(x)+\varepsilon N L_{1}(x) \\
& \approx N L\left(x_{i}\right)+\beta\left(\frac{d N L(X)}{d x}\right)_{x_{i}}^{T}\left(x-x_{i}\right)^{\prime} \quad \text { with } 0 \leq \beta \leq 1 .
\end{aligned}
$$

Hence, the linearization around $x_{i}$ of (6) $x_{i}$ lead to

$x_{k+1}=A_{i, k} x_{k}+B_{i, k} U_{k}+C_{i, k}, i=1, \ldots, S ; k=0, \ldots, N-1$

where $A_{i, k}$ and $B_{i, k}$ are square matrices of $N \times N$ order and $C_{i, k}$ a matrix of $N \times 1$ order. Therefore, the optimal control problem (5)-(6) becomes a linear quadratic problem. The feedback control solution is given by the following expression [19]:

$U_{i, k}=-K_{i} x_{k}, i=1, \ldots, S ; k=0, \ldots, N-1$,

where

$K_{i}=\left(Q+B_{i}^{T} E_{i} B_{i}\right)^{-1} B_{i}^{T} E_{i} A_{i}$,

is the feedback gain matrix and $E_{i}$ denotes discrete Riccati equation solution of the following form

$E_{i}-Q-A_{i}^{T} E_{i} A_{i}+A_{i}^{T} E_{i} B_{i}\left(R+B_{i}^{T} E_{i} B_{i}\right)^{-1} B_{i}^{T} E_{i} A_{i}=0$. 
It is obvious to realise that the linearization around every operating point gives the system for which the equations have the form (10). Since there is $S$ operating points, we obtain $S$ systems. Consequently, we have $S$ controls calculated using (11). To get only one system and only one control $U_{k}$, we use defuzzyfication method [20]. Therefore, using (10) this transformation gives the following equation

$x_{k+1}=A x_{k}+B U_{k}+C, k=0, \ldots, N-1$,

$U_{k}=-K x_{k}, k=0, \ldots, N$,

where

$$
A=\frac{\sum_{i=1}^{S} \omega_{i}\left(x_{i}\right) A_{i, k}}{\sum_{i=1}^{S} \omega_{i}\left(x_{i}\right)}, \quad B=\frac{\sum_{i=1}^{S} \omega_{i}\left(x_{i}\right) B_{i, k}}{\sum_{i=1}^{S} \omega_{i}\left(x_{i}\right)} \quad C=\frac{\sum_{i=1}^{S} \omega_{i}\left(x_{i}\right) C_{i, k}}{\sum_{i=1}^{S} \omega_{i}\left(x_{i}\right)} \text {, and } K=\frac{\sum_{i=1}^{S} \omega_{i}\left(x_{i}\right) K_{i, k}}{\sum_{i=1}^{S} \omega_{i}\left(x_{i}\right)},
$$

and $w_{i}\left(x_{i}\right)$ denotes membership degree corresponding to the operating point $x_{i}$.

\section{Discretization optimal control problem}

We use two different methods to approximate the optimal control problem (4), (1)-(3): Fuzzy logic strategy and direct method. The purpose is two compare the numerical solution in order to conclude from the evidence the efficacy of fuzzy logic strategy.

\subsection{Fuzzy logic strategy use}

Setting

$X=\left(T-T_{0}, I, V\right)^{t}$ and $U=\left(u_{1}(t), u_{2}(t)\right)^{t}$.

the system (1)-(3) becomes

$$
\left\{\begin{array}{l}
\frac{d X_{1}}{d t}=s-q\left(X_{1}+T_{0}\right)+a\left(X_{1}+T_{0}\right)\left[1-\frac{X_{1}+T_{0}}{T_{\max }}\right]-\beta e^{-U_{1}} \frac{\left(X_{1}+T_{0}\right) X_{3}}{1+b X_{3}}, \\
\frac{d X_{2}}{d t}=\beta e^{-I I_{1}} \frac{\left(X_{1}+T_{0}\right) X_{3}}{1+b X_{3}}-\delta X_{2}, \\
\frac{d X_{3}}{d t}=p e^{-t J_{2}} X_{2}-c X_{3} .
\end{array}\right.
$$


For $\mathrm{k}=0, \ldots, \mathrm{N}$, using forward explicit Euler scheme to approximate the mathematical model (1)(3), we obtain

$$
\left\{\begin{aligned}
X_{1, k+1}= & (1+h a-h q) X_{1, k}+h\left(s-q T_{0}\right)+h a\left[-X_{1, k} \frac{\left(X_{1, k}+T_{0}\right)}{T_{\max }}+T_{0}\left(1-\frac{\left(X_{1, k}+T_{0}\right)}{T_{\max }}\right)\right], \\
& +\beta h\left(\frac{\left(X_{1, k}+T_{0}\right) X_{3, k}}{1+b X_{3, k}}\right) U_{1}-\beta h \frac{\left(X_{1, k}+T_{0}\right) X_{3, k}}{1+b X_{3, k}}, \\
X_{2, k+1}= & (1-h \delta) X_{2, k}-h \beta\left(\frac{\left(X_{1, k}+T_{0}\right) X_{3, k}}{1+b X_{3, k}}\right) U_{1}+h \beta \frac{\left(X_{1, k}+T_{0}\right) X_{3, k}}{1+b X_{3, k}}, \\
X_{3, k+1}= & (1-h c) X_{3, k}-h p X_{2, k} U_{2}+h p X_{2, k},
\end{aligned}\right.
$$

where $h=\frac{T_{N}}{N}, X_{k}=X\left(t_{k}\right)$ and we have set $e^{z} \approx 1+z, \forall z \in R$.

Let $T_{i}, \quad I_{i}$ and $V_{i}, \quad i=1,2,3$ be the first, second and third operating points that take values in the labels centers of a universe of discourse $X$. Then (18) can be rewritten as follows

$$
\left\{\begin{array}{rl}
X_{1, k+1}= & (1+h a-h q) X_{1, k}+h\left(s-q T_{0}\right)+h a\left[-T_{i} \frac{\left(T_{i}+T_{0}\right)}{T_{\max }}+T_{0}\left(1-\frac{\left(T_{i}+T_{0}\right)}{T_{\max }}\right)\right] \\
& +\beta h\left(\frac{\left(T_{i}+T_{0}\right) V_{i}}{1+b V_{i}}\right) U_{1}-\beta h \frac{\left(T_{i}+T_{0}\right) V_{i}}{1+b V_{i}} \\
X_{2, k+1}= & (1-h \delta) I_{i}-h \beta\left(\frac{\left(T_{i}+T_{0}\right) V_{i}}{1+b V_{i}}\right) U_{1}+h \beta \frac{\left(T_{i}+T_{0}\right) V_{i}}{1+b V_{i}} \\
X_{3, k+1}= & (1-h c) V_{i}-h p I_{i} U_{2}+h p I_{i}
\end{array}, i=1,2,3\right.
$$

and setting $X_{k}=\left(X_{1, k}, X_{2, k}, X_{3, k}\right)^{t}$ its compact form becomes

$X_{k+1, i}=A_{s} X_{k, i}+B_{i} U_{k, i}+C_{i}, \quad i=1,2,3$,

where

$$
\begin{gathered}
A_{i}=\left(\begin{array}{ccc}
1+h a-h q & 0 & 0 \\
0 & 1-h \delta & 0 \\
0 & 0 & 1-c h
\end{array}\right), \\
B_{i}=h\left(\begin{array}{cc}
\beta \frac{\left(T_{i}+T_{0}\right) V_{i}}{1+b V_{i}} & 0 \\
-\beta \frac{\left(T_{i}+T_{0}\right) V_{i}}{1+b V_{i}} & 0 \\
0 & -p I_{i}
\end{array}\right), i=1,2,3
\end{gathered}
$$


and

$$
C_{i}=h\left(\begin{array}{l}
-a T_{i} \frac{\left(T_{i}+T_{0}\right)}{T_{\max }}+a T_{0}\left[1-\frac{\left(T_{i}+T_{0}\right)}{T_{\max }}\right]-\beta \frac{\left(T_{i}+T_{0}\right) V_{i}}{1+b V_{i}}+\left(s-q T_{0}\right) \\
\beta \frac{\left(T_{i}+T_{0}\right) V_{i}}{1+b V_{i}}, \\
p I_{i},
\end{array}\right), i=1,2,3 .
$$

Using the rectangular rule, the cost functional (4) gives the following form

$J^{N}(X, U)=\sum_{k=0}^{N-1}\left(X_{k}^{T} R_{1} X_{k}+U_{k}^{T} R_{2} U_{k}\right) h$,

where for elements $\mathrm{a}_{1}, \mathrm{a}_{2}$ and $\mathrm{a}_{3}$ and $\mathrm{b}_{1}, \mathrm{~b}_{2} R_{1}$ and $R_{2}$ are matrices defined as follows

$$
R_{1}=\left(\begin{array}{ccc}
a_{1} & 0 & 0 \\
0 & a_{2} & 0 \\
0 & 0 & a 3
\end{array}\right), \quad R_{2}=\left(\begin{array}{cc}
b_{1} & 0 \\
0 & b_{2}
\end{array}\right)
$$

where $\mathrm{a}_{1}, \mathrm{a}_{2}, \mathrm{a}_{3}$ and $\mathrm{b}_{1}, \mathrm{~b}_{2}$ are constant elements of corresponding matrix.

Therefore, the solution of optimal control problem (4), (1)-(3) using fuzzy logic is obtain by determining $U^{*}=\left(U_{0}^{*}, \ldots, U_{N-1}^{*}\right)^{T}$ solution of (24) subject to (20). This problem is a linear quadratic (LQ) of three linear state systems whose solution leads to three feedback controls of the form

$$
U_{k}=K_{i} X_{k}, \quad i=1,2,3 ; \quad k=0, \cdots, N,
$$

where $\mathrm{K}_{\mathrm{i}}$ is a gain feedback.

After this discretization of the optimal control problem (4), (1)-(3) using fuzzy logic strategy, we focus now on its approximation that uses direct approach.

\subsection{Direct approach use}

Let $B^{N}=\left\{\psi_{j}^{N}, j=1, \ldots, N\right\}$ be a set of linear B-splines basis functions on the uniform grid 
$\Omega_{N}=\left\{t_{k}=\frac{{ }_{\mathrm{k}} T_{N}}{N},\{k=0, \ldots, N\}\right\}$.

The direct method consists of determining the discrete form of state equations (1)-(3) and the cost functional (4) using linear B-splines functions $B^{\mathrm{N}}$. The function $\psi_{\mathrm{i}}^{\mathrm{N}}$ are defined such that $\psi_{i}^{N}\left(t_{k}\right)=\delta_{i k}$,

where $\delta_{\text {ik }}$ denotes Kronecker symbol. Let $W^{N}$ be a vector space whose the basis is $B^{N}$ satisfying

$\operatorname{dim} W^{N}=N \quad$ and $W^{N} \subset W^{N+1}$.

We consider $\mathrm{W}=\mathrm{C}^{0}\left(0, \mathrm{~T}_{\mathrm{N}}\right)$ and we take the interpolation operator

$$
\begin{aligned}
& \Pi^{N}: W \rightarrow W^{N} \\
& \varphi \mapsto \Pi^{N} \varphi
\end{aligned}
$$

satisfying

$\Pi^{N} \varphi\left(t_{k}\right)=\varphi\left(t_{k}\right), \quad k=1, \cdots, N$.

Therefore, system (1)-(3) is approximated using $\Pi^{N}$ in $\mathrm{W}^{\mathrm{N}}$ to the following form

$\frac{d T^{N}}{d t}=s-q T^{N}+a T^{N}\left[1-\frac{T^{N}}{T_{\max }}\right]-\beta^{-u_{1}} \frac{T^{N} V^{N}}{1+b V^{N}}$

$\frac{d I^{N}}{d t}=\beta e^{-u_{1}} \frac{T^{N} V^{N}}{1+b V^{N}}-\delta I^{N}$,

$\frac{d V^{N}}{d t}=p e^{-u_{2}} I^{N}-c V^{N}$

$T^{N}(0)=T^{N, 0}, \quad I^{N}(0)=I^{N, 0}, \quad V^{N}(0)=V^{N, 0}$.

Setting $\lambda=\left(u_{1}, u_{2}\right)^{t}$ and $q=\left(q_{u_{1}}, q_{u_{2}}\right)^{t}$, the discretization of the cost functional (4) is

$J^{N}\left(\lambda^{M}\right) \approx \sum^{k=1}\left(q_{T}\left(T^{N}\left(t_{k}\right)-T_{0}\right)^{2}+\sum_{j}^{j=1} q_{j}\left(\lambda_{j, k}^{M}\right)^{2}\right) h, \quad$ with $h=\frac{T_{\max }}{M}$

which can be rewritten in compact form as follows

$J^{N}\left(\underline{\lambda}^{M}\right) \approx h\left(\left(Y^{T} R Y\right)+\left(\underline{\lambda}^{M}\right)^{T} B \underline{\lambda}^{M}\right)$ 
where $\underline{\lambda}^{M}$ is an $(M+1) \times 2$ matrix whose elements $\lambda_{j, k}^{M}$ are components of $\lambda_{j}^{N}$ in the set $B^{N}, Y$ represents the matrix whose $k^{t h}$ component $T^{N}\left(t_{k}\right)-T_{0}$ is associated to $\lambda=\lambda^{N}$ $=\hat{\mathbf{u}}_{1}^{N}, u_{2}^{N} \boldsymbol{\vartheta}, R$ and $B$ are matrix defined by $R=\left(\begin{array}{cc}q_{T} & 0 \\ 0 & q_{T}\end{array}\right), \quad B=\left(\begin{array}{cc}q_{u_{1}} & 0 \\ 0 & q_{u_{2}}\end{array}\right)$.

Finally, using direct method we find $\lambda^{*, M} \in R^{(M+1)} \times R^{(M+1)}$ that minimizes (35) subject to (30)-(33).

\section{Numerical test}

The role of therapy prescribed for HBV is to remove all infected hepatocytes and virions in the body of patient to allow uninfected hepatocytes to be the equilibrium value $T_{0}$. We consider a healthy subject with $T_{0}=1000$ cells/dl, $I=0$ cells/dl and $V=0$ cells/dl [12]. To use fuzzy logic strategy, we consider the universe of discourse $X$ with three linguistic variables: uninfected hepatocytes (UH), infected hepatocytes $(\mathrm{IH})$ and free virions (FV), respectively. According to physiology [7], [18], we consider $T \in[200,1500], \quad I \in[0,300]$ and $V \in[0,500]$. Each variable has three states: low, middle and high. It is taken as low, middle and high if its value is less than the lower bound of interval, in the interval and greater than upper bound of interval, respectively. Then, LUH (Low uninfected hepatocytes), NUH (Normal uninfected hepatocytes) and GUH (the highest uninfected hepatocytes) are terms (fuzzy sets) of the linguistic variable UH. Similarly, LIH, NIH and GUH (respectively LFV, NFV and GFV) are used as terms of the variable linguistic IH (respectively FV). From the relation (17), we have $X_{1} \in[-800,500], X_{2} \in[0,300]$ and $X_{3} \in[0,500]$. In the universe of discourse $X$ the status of linguistic variables is understood in such way that we consider the labels centered respectively at $-800,-150$ and 500 for $\mathrm{UH}$; at 0,150 and 300 for $\mathrm{IH}$ and at 0,250 and 500 for FV. Hence we suppose that theses centers constitute the operating points values of the system (18). Below Table1 shows operating points associated to those linguistic variables whereas membership functions associated to this labeling are represented in the Figure 1, 2 and 3, respectively. 


\begin{tabular}{|c|c|}
\hline Variable & Operating Points \\
\hline $\mathrm{T}$ & {$[-800 ; 150 ; 500]$} \\
\hline $\mathrm{I}$ & {$[0 ; 150 ; 300]$} \\
\hline $\mathrm{V}$ & {$[0 ; 250 ; 500]$} \\
\hline
\end{tabular}

Table 1: Variables and their operating points

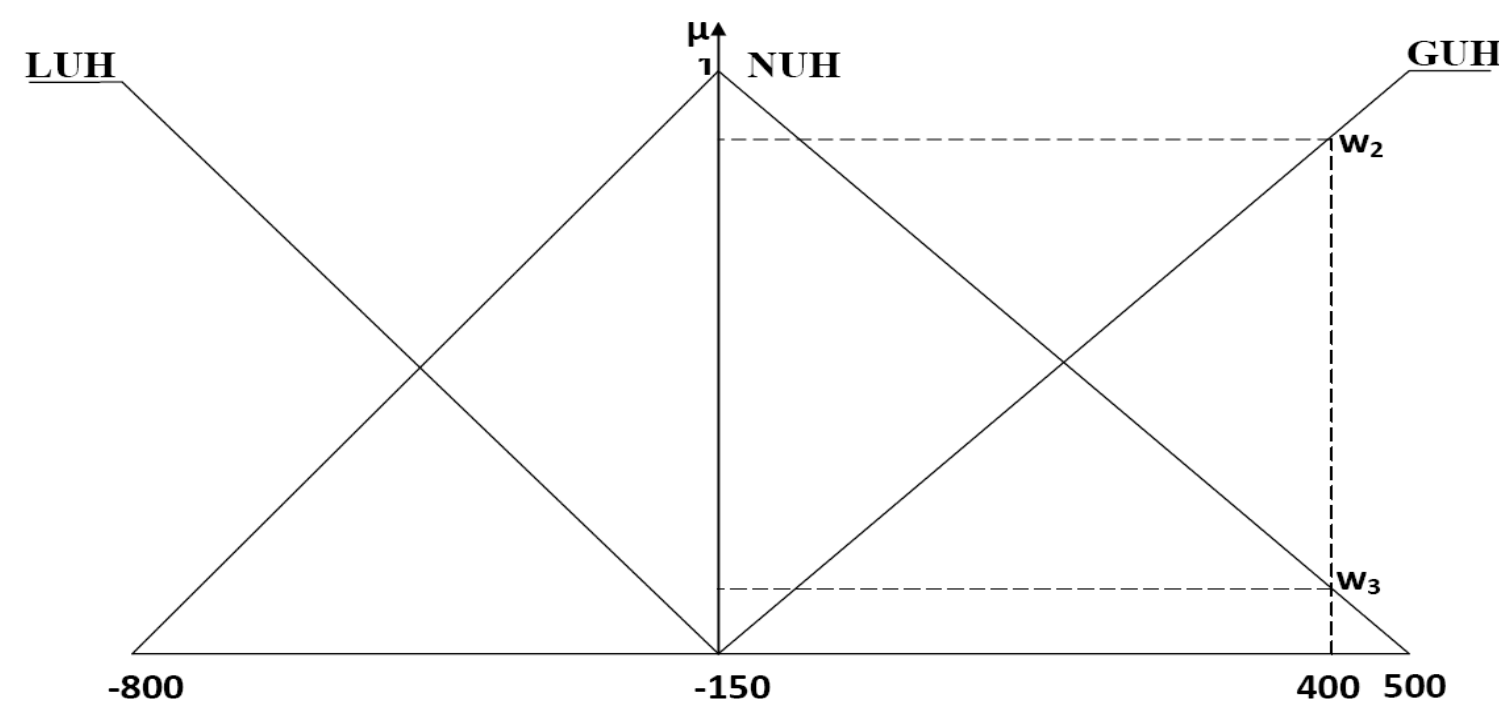

Figure 1: Membership function for uninfected hepatocytes

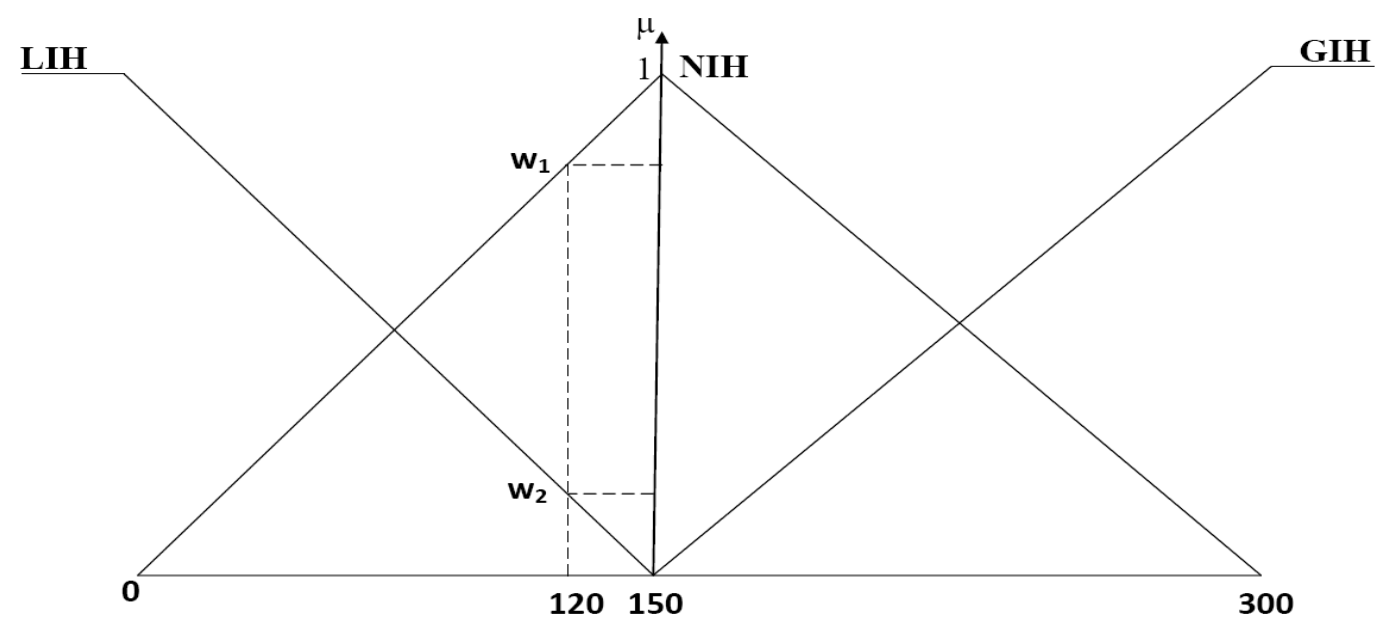

Figure 2: Membership function for infected hepatocytes 


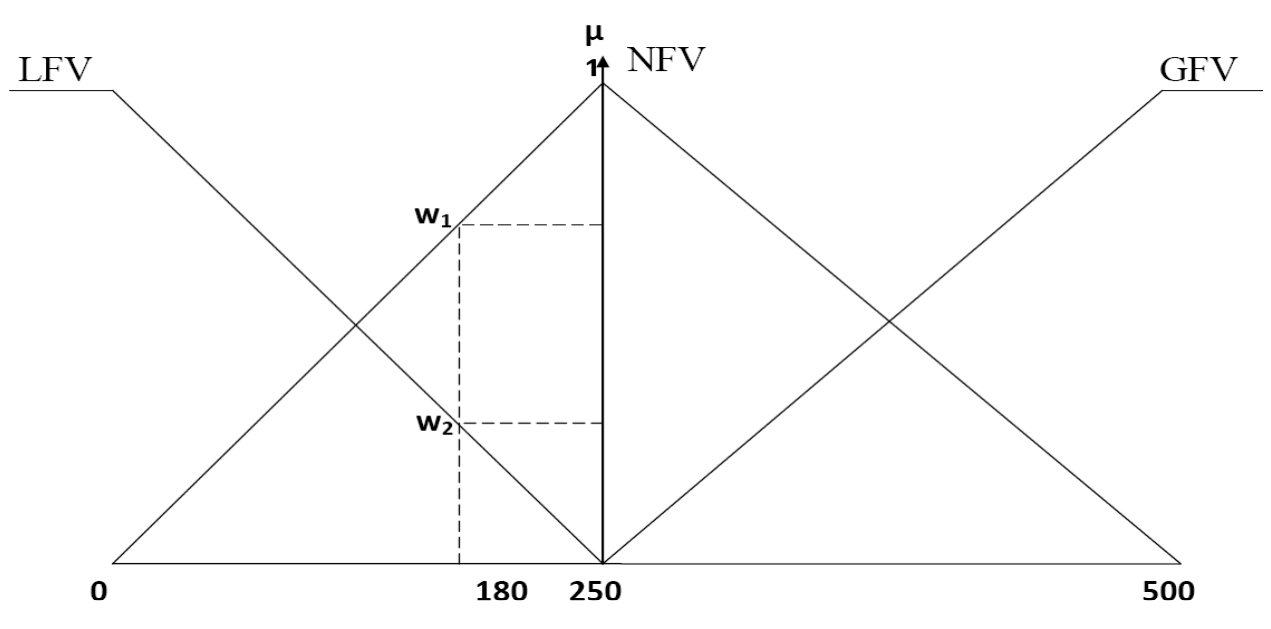

Figure 3: Membership function for free virions

The values of parameters used in numerical simulation are presented in Table 2 [12].

\begin{tabular}{|l|c|c|c|c|c|c|c|c|c|}
\hline Parameter & $\mathrm{a}$ & $\mathrm{q}$ & $\mathrm{s}$ & $\beta$ & $\mathrm{c}$ & $\mathrm{p}$ & $\mathrm{b}$ & $\delta$ & Tmax \\
\hline Value & 0.108 & 0.072 & 36 & 0.001 & 3 & 5 & 0.01 & 0.5 & 1500 \\
\hline
\end{tabular}

Table 2: Parameters used in numerical simulation

Using values $N=100$ and $\mathrm{TN}=12$ (We consider also a patient under treatment during $\mathrm{TN}=12$ months) the relations (21), (22) and (23) give after calculations the following matrices

$$
\begin{aligned}
A_{i} & =\left(\begin{array}{ccc}
1.0036 & 0 & 0 \\
0 & 0.95 & 0 \\
0 & 0 & 0.7
\end{array}\right), i=1,2,3 \\
B_{1} & =\left(\begin{array}{ll}
0 & 0 \\
0 & 0 \\
0 & 0
\end{array}\right), B_{2}=\left(\begin{array}{cc}
6.0714 & 0 \\
-6.0714 & 0 \\
0 & -75
\end{array}\right), B_{3}=\left(\begin{array}{cc}
12.5 & 0 \\
-12.5 & 0 \\
0 & -150
\end{array}\right), \\
C_{1} & =\left(\begin{array}{c}
6.9120 \\
0 \\
0
\end{array}\right), C_{2}=\left(\begin{array}{c}
-4.0734 \\
6.0714 \\
75
\end{array}\right), C_{3}=\left(\begin{array}{c}
-21.5 \\
12.5 \\
150
\end{array}\right) .
\end{aligned}
$$

We consider that $R_{1}$ and $R_{2}$ are identity matrices and using (26) we obtain 


$$
\begin{gathered}
K_{1}=\left(\begin{array}{lll}
0 & 0 & 0 \\
0 & 0 & 0
\end{array}\right), K_{2}=\left(\begin{array}{ccc}
0.0815 & -0.0772 & 0 \\
0 & 0 & -0.0093
\end{array}\right) \\
K_{3}=\left(\begin{array}{ccc}
0.0400 & -0.0379 & 0 \\
0 & 0 & -0.0047
\end{array}\right) .
\end{gathered}
$$

The defuzzification transformation concerns rows of matrixes $A_{i}, B_{i}, C_{i}$ and $K_{i}, i=1,2,3$ using the relation (16). Furthermore, we consider $\omega_{i}, i=1,2,3$ degree of membership for each membership function. Let 400 cells/dl, 120 cells/dl and 180 cells/dl be entry of uninfected hepatocytes, infected hepatocytes and free virions respectively. After calculations of degrees of membership of each linguistic $\mathrm{T}$, I and $\mathrm{V}$ that is the values of $\mathrm{w} 1$, w2 and $\mathrm{w} 3$ from the membership functions illustrated in the Figures 1, 3 and 3, the results are shown in the Table 3.

\begin{tabular}{|c|c|c|c|}
\hline Variable & $\mathbf{w}_{\mathbf{1}}$ & $\mathbf{w}_{\mathbf{2}}$ & $\mathbf{W}_{\mathbf{3}}$ \\
\hline $\mathrm{T}$ & 0 & 0.85 & 0.15 \\
\hline $\mathrm{I}$ & 0.8 & 0.2 & 0 \\
\hline $\mathrm{V}$ & 0.72 & 0.28 & 0 \\
\hline
\end{tabular}

Table 3: Variables and their corresponding degrees of membership

Hence the following matrices are calculated using defuzzification transformations, that is the formula (16).

$$
\begin{gathered}
A=\left(\begin{array}{ccc}
1.0036 & 0 & 0 \\
0 & 0.95 & 0 \\
0 & 0 & 0.7
\end{array}\right), B=\left(\begin{array}{cc}
7.0357 & 0 \\
-1.2143 & 0 \\
0 & -21
\end{array}\right) \quad C=\left(\begin{array}{c}
-6.6874 \\
1.2143 \\
21
\end{array}\right) \\
K=\left(\begin{array}{ccc}
0.0753 & -0.0753 & 0 \\
0 & 0 & -0.0019
\end{array}\right) .
\end{gathered}
$$

Below, Figures 4 and 5 show the results from the numerical simulations of a patient suffering from HBV under treatment prescribed during a period of 12 months. 


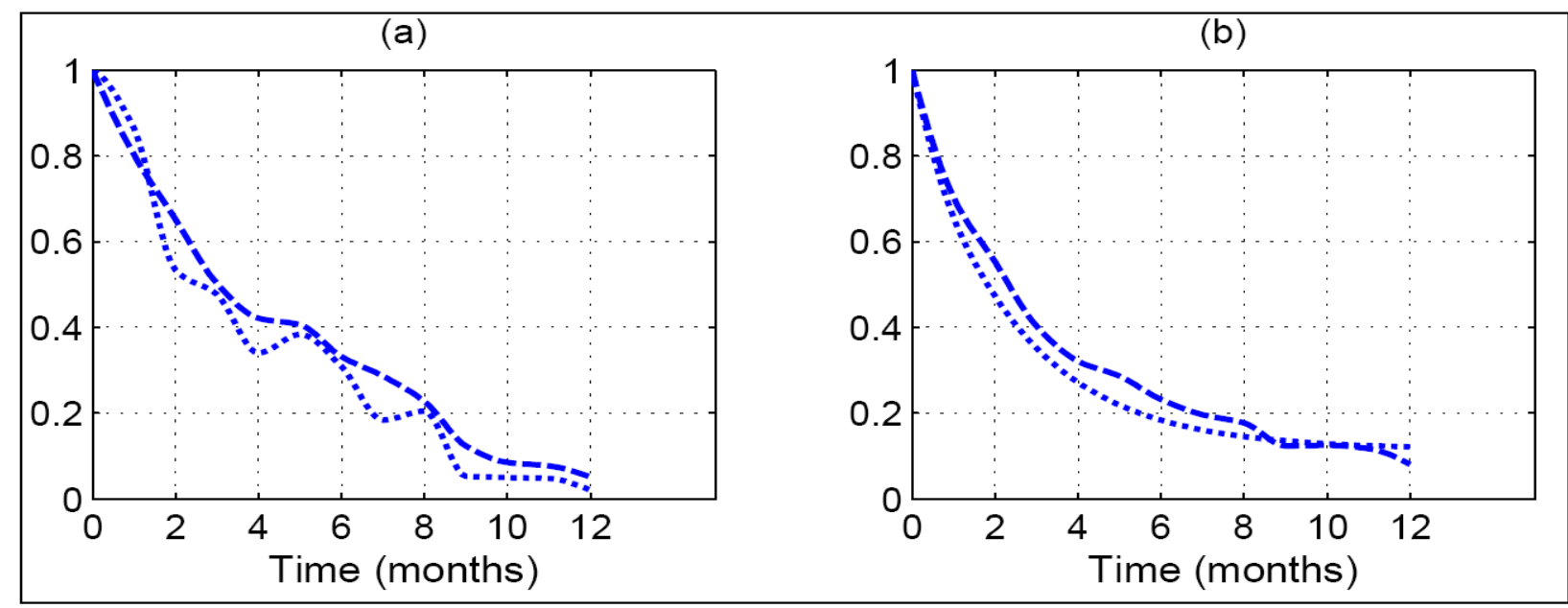

Figure 4: Variation of drugs (control) $u_{1}$ (a) and $u_{2}$ (b).

In this figure, the curves in dotted line represent the parameter using direct approach whereas the curves in dashed line show the parameter using fuzzy logic strategy. Figure 4 illustrates both chemotherapy $u_{1}$ and $u_{2}$ prescribed for a patient suffering from HBV. The control $u_{1}$ is imposed to prevent the virus to infect the hepatocytes cells whereas the role of control $u_{2}$ is to prevent infected hepatocytes cells to produce the new viruses. We consider that the number 1 stands for absence of treatment and 0 means the patient is at the end of this treatment (maximal use of therapy). This assumption is used in numerical simulation and we realize that the Figure 4 shows a decrease of controls in number from 1 to be closer to the lower value 0 . This means that patient is not suffering from the effects of $\mathrm{HBV}$ while he/she is under treatment. 


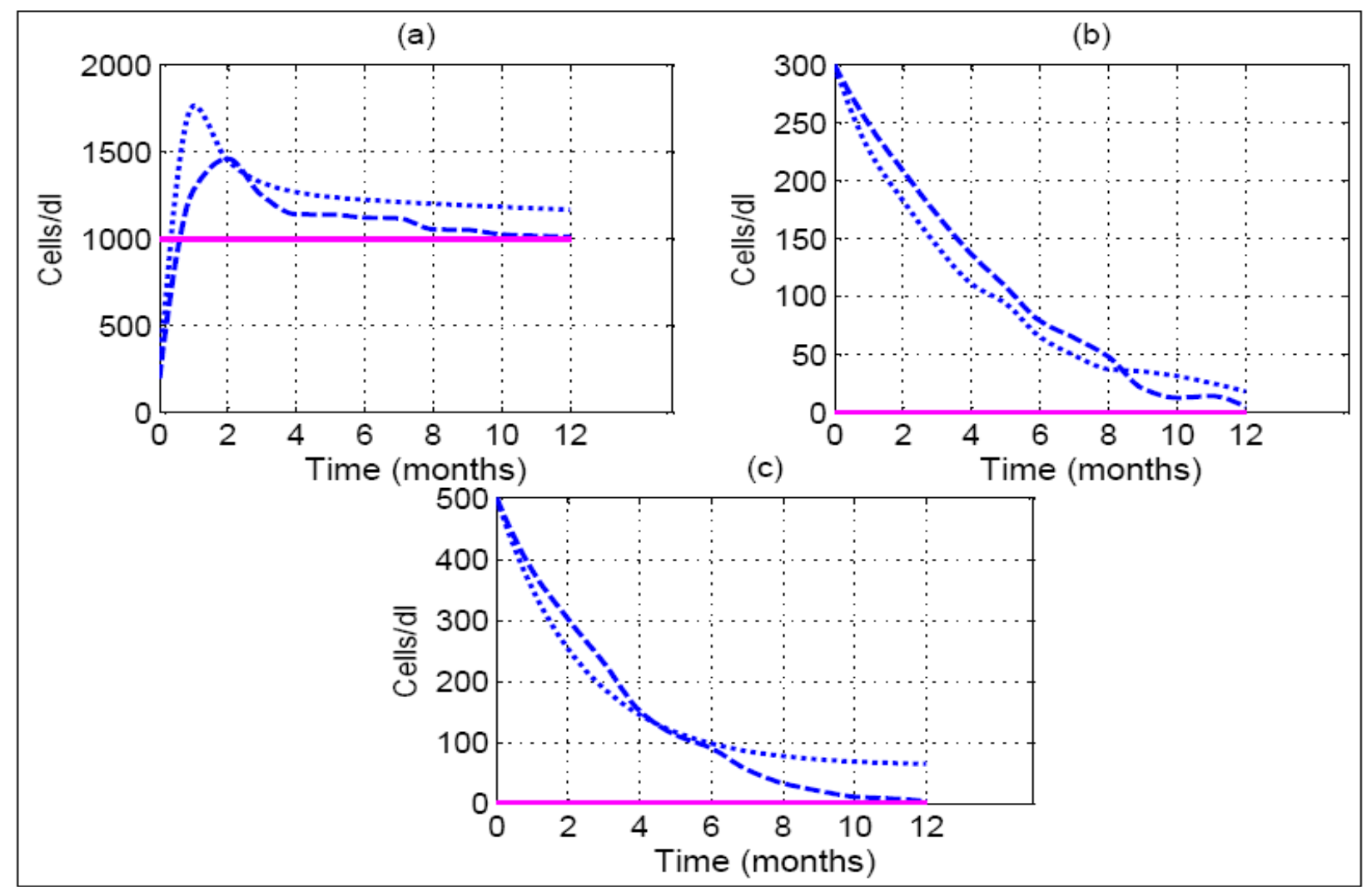

Figure 5: Variation of the concentration of uninfected hepatocytes (a), infected hepatocytes (b) and free virions (c) for a patient suffering from HBV under treatment during a period 12 months. The curves in dotted line represent the parameter using direct approach whereas the curves in dashed line show the parameter using fuzzy logic strategy.

The Figure 5 shows the response of these controls to variation of the concentration of uninfected hepatocytes (Figure 5(a)), infected hepatocytes (Figure 5(b)) and free virions (Figure 5(c)) respectively. The Figure (a) shows an increase of uninfected hepatocytes to its higher value during the first month of treatment and they decrease to reach the equilibrium value for healthy subject. The Figures 5(b) and 5(c) illustrate that both infected hepatocytes and free virions decrease from starting time of taking therapeutic drugs to reach their equilibrium values for healthy subject.

\section{Concluding remarks}

In the current paper, an optimal control problem related to HBV dynamics has been exposed and solved with the use of two different numerical methods, i.e direct and fuzzy logic methods. In this context, we found the optimal trajectories of uninfected hepatocytes, infected hepatocytes 
and free virions as response to two controls of hepatitis B virus. These controls are drugs prescribed for a patient suffering from HBV. The findings show that the results obtained using fuzzy logic strategy are satisfactory and provide the curves which are close to those given by numerical simulation using standard method known as direct method. Therefore, this justifies the efficacy of fuzzy logic strategy in solving optimal control problems.

\section{References}

[1] R. M. Anderson and R. M. May, Infectious Disease of Humans: Dynamics and Control, Oxford University Press, Oxford, UK, 1991

[2] S. Bhattacharyya and S. Ghosh, Optimal control of vertically transmitted disease, Computational and Mathematica 1Methods in Medicine, vol. 11, no. 4, pp. 369-387, 2010.

[3] J. Danane and K. Allali, Mathematical Analysis and Treatment for a Delayed Hepatitis B Viral Infection Model with the Adaptive Immune Response and DNA-Containing Capsids, High-Throughput 2018, 7, 35; doi:10.3390/ht7040035

[4] M. Gatto, S. Muratori and S. Rinaldi, A functional interpretation of the logistic equation, Ecological modelling, Volume 42, Issue 2, August 1988, Pages 155-159

[5] A. M. Elaiw, M. A. Alghamdi and Shaban Aly, Hepatitis B Virus Dynamics: Modeling, Analysis, and Optimal Treatment Scheduling, Hindawi Publishing Corporation 2013, Discrete Dynamics in Nature and Society, Volume 2013, Article ID 712829, 9 pages, http://dx.doi.org/10.1155/2013/712829

[6] A. R McLean, B. S Blumberg. Modeling the impact of mass vaccination against hepatitis B. I. Model formulation and parameter estimation. Proc Biol Sci. 1994;256:7-15.

[7] S. Kadelka and S. M Ciupe, Mathematical investigation of HBeAg seroclearance, Mathematical Biosciences and Engineers, 16(6): 7616-7658.

[8] T. K. Kar and A. Batabyal, Stability analysis and optimal control of an SIR epidemic model with vaccination, Biosystems, vol. 104, no. 2-3, pp. 127-135, 2011.

[9] T. K. Kar and S. Jana, A theoretical study on mathematical modelling of an infectious disease with application of optimal control, Biosystems, vol. 111, no. 1, pp. 37--50, 2013.

[10] N. Krichen Masmoudi and N. Derbel, Optimal control of nonlinear systems by fuzzy logic, ISCIII, Nabeul, Tunisia, 2003. 
[11] Kwakernaak,. R. Savan, Linear. Optimal. Control. Systems,. Wiley. Inter-Science,. New. York,. 1972.

[12] S. Lenhart and J. T. Workman, Optimal Control Applied to Biological Models, Mathematical and Computational Biology Series, Chapman \& Hall/CRC, London, UK, 2007.

[13] P. Liang, J. Zu and G. Zhuang, A Literature Review of Mathematical Models of Hepatitis B Virus Transmission Applied to Immunization Strategies from 1994 to 2015, Journal of Epidemiology, 2018;28(5):221-229

[14] J. M. Ntaganda, S. Ousséni, G. Barro and B. Mampassi, Using fuzzy logic strategy for solving a therapeutic optimal control problem of an HIV dynamical infection, Journal of Pure and Applied Mathematics: Advances and Applications Volume 3, Number 2, 2010, Pages 205-234

[15] J. M. Ntaganda, M. S. Daoussa Haggar, B. Mampassi: Fuzzy Logic Strategy for Solving an Optimal Control Problem of Therapeutic Hepatitis C Virus Dynamics” Open Journal of Applied Sciences, 2015, 5, 527-541

[16] J. Pang, J. A. Cui, and X. Zhou, Dynamical behavior of a hepatitis B virus transmission model with vaccination, Journal of Theoretical Biology, vol. 265, no. 4, pp. 572--578, 2010.

[17] M. I. Park, E. Kim, S .Ji and M. Park, A new approach to fuzzy modeling, IEEE Trans. Fuzzy Syst, vol. 5, no. 3, pp. 328-337, August 1987.

[18] S. Pungpapong, W. Ray Kim, and J. J. Poterucha, Natural History of Hepatitis B Virus Infection: An Update for Clinicians, Mayo Clin Proc. 2007;82(8):967-975

[19] M. Sugeno et K. Murakami, Fuzzy parking control of model car, in 23rd IEEE Conf. on Decision and Control, 1984.

[20] T. Takagi and M. Sugeno, Fuzzy Identification of Systems and its Applications to modeling and Control, IEEE Trans. Systems Man And Cybernetics, vol. 15, pp. 116-132, 1985.

[21] World Health Organization, Hepatitis B Fact Sheet No. 204, The World Health Organisation, Geneva, Switzerland, 2013, http://www.who.int/ mediacentre/factsheets/fs204/en/.

[22] World Health Organization, Hepatitis B.Fact Sheet, World Health Organization, Revised 
July 2017. Available online at: http://www.who.int/mediacentre/factsheets/fs204/en/

[23] S. J. Yakowitz, The Stagewise Kuhn-Tucker Condition and Differential Dynamic Programming, IEEE Trans, vol.31, $\mathrm{N} \circ 1$, pp. 25-30, 1986

[24] S. J. Zhao, Z.Y. Xu, and Y. Lu, A mathematical model of hepatitis B virus transmission and its application for vaccination strategy in China, International Journal of Epidemiology, vol. 29, no. 4, pp. 744-752, 2000. 